Vietnam Journal of Mechanics, VAST, Vol. 31, No. 1 (2009), pp. 1-16

\title{
EXTENDED AVERAGED EQUATION METHOD AND APPLICATION ON ANALYZING SOME NONLINEAR DETERMINISTIC VIBRATIONS
}

\author{
${ }^{1}$ Nguyen Dong Anh, ${ }^{2}$ Ninh Quang Hai, ${ }^{3}$ Werner Schiehlen \\ ${ }^{1}$ Institute of mechanics, Hanoi, Vietnam, \\ ${ }^{2}$ Architectural University, Hanoi, Vietnam \\ ${ }^{3}$ InstituteBof Mechanics, StuttgartUniversity, Germany
}

\begin{abstract}
The paper presents an extended averaged equation approach to the investigation of nonlinear vibration problems. The proposed method is applied to some free/selfexcited oscillators, nonlinear free and forced oscillations of a suspension system with two-degree-of-freedom. The results in analyzing the vibration systems with different nonlinearity show the efficiency and advantages of the method.
\end{abstract}

Keywords: free oscillation, self-excited oscillation, oscillation of a suspension system.

\section{INTRODUCTION}

All real engineering systems are nonlinear and subject to excitation during their operation. Research on vibration phenomena in nonlinear systems with an aim to reduce undesired vibration is needed. A great interest to researchers is to develop new methods for investigating nonlinear vibrations preferably applicable to wide classes of nonlinear systems including weak and strong nonlinearity, subject to deterministic and/or random excitations [1-9].

The method of moment equation is well known for analysis of random nonlinear vibration phenomena and gives also good approximate solutions for systems with strong nonlinearity [10-11]. The question is whether the method can be extended to deterministic systems. A proposed approach given in this paper concerned with some following classical methods. The averaging method is attributed to Bogoliubov and Mitropolsky [2]. The method, however, dates from the 18th century and was proved first to be correct by Fatou. More up-to-date references for the averaging method were given in [15, 16]. An extension of averaging method to nonlinear to strongly nonlinear oscillations is proposed in [17]. The idea of putting the nonlinear system in the form of the extended equation was proposed by Povzner in 1974, see also the book by Bogaevsky and Povzner [18]. In the paper, an extended averaged equation for deterministic systems is presented and then, for illustration, some free/ self-excited oscillators and nonlinear free/ forced oscillations of a suspension system with two-degree-of-freedom [21 - 22] are investigated. 


\section{EXTENDED AVERAGED EQUATION FOR DETERMINISTIC SYSTEM}

Consider a nonlinear system

$$
\dot{Z}=F(Z)+P \cos \nu t, \quad Z=\left(z_{1}, z_{2}, \ldots, z_{n}\right)^{T},
$$

where, $F(Z)=\left(F_{1}(Z), F_{2}(Z), \ldots, F_{n}(Z)\right)^{T}$ is a nonlinear vector function of the vector $Z$, $\mathrm{P}=\left[p_{1}, p_{2}, \ldots, p_{n}\right]^{T}$ is a constant vector. It is assumed that there exists a corresponding linear system

$$
\dot{X}=A X, \quad X=\left(x_{1}, x_{2}, \ldots, x_{n}\right)^{T}, A=\left\{a_{i j}\right\} \quad(i, j=1,2, \ldots, n),
$$

where $A$ is a constant matrix. The term "corresponding" means the nonlinear and linear systems have the same dimension, i.e, the numbers of components of the vectors $Z$ and $X$ are equal to each other. Furthermore, the linear system should have expected properties of the nonlinear system. For example, if we are interested in periodic solutions of the nonlinear system then the corresponding linear system should be taken in the form that can possess them too. In general, the matrix A is fully unknown; however, if some properties of the nonlinear system responses are prior known then the matrix A should be choosen in such a way to provide those properties.

For an arbitrary differentiable function $\Psi(t, Z, X)$ one gets

$$
\frac{d \Psi}{d t}=\frac{\partial \Psi}{\partial t}+\sum_{i=1}^{n} \frac{\partial \Psi}{\partial z_{i}}\left(F_{i}(Z)+p_{i} \cos \nu t\right)+\sum_{i=1}^{n} \frac{\partial \Psi}{\partial x_{i}}\left(\sum_{j=1}^{n} a_{i j} x_{j}\right) .
$$

Denote the averaging operator as

$$
<.>=\lim _{T \rightarrow+\infty} \frac{1}{T} \int_{0}^{T}(.) d t .
$$

Suppose that the averaged value of $\Psi$ doesn't depend on the time

$$
\langle\Psi\rangle=\text { const. }
$$

It follows

Thus, one gets

$$
\left\langle\frac{d \Psi}{d t}\right\rangle=\frac{d}{d t}\langle\Psi\rangle=0,\left\langle\frac{\partial \Psi}{\partial t}\right\rangle=\frac{\partial}{\partial t}\langle\Psi\rangle=0 .
$$

$$
\sum_{i=1}^{n}\left\langle\frac{\partial \Psi}{\partial z_{i}}\left(F_{i}(Z)+p_{i} \cos \nu t\right)\right\rangle+\sum_{i=1}^{n}\left\langle\frac{\partial \Psi}{\partial x_{i}}\left(\sum_{j=1}^{n} a_{i j} x_{j}\right)\right\rangle=0 .
$$

Here, we consider functions of the form $\Psi=r_{k s}(t) z_{k}^{m} z_{s}^{p}, \Psi=g_{k s}(t) x_{k}^{m} x_{s}^{p}$ and $\Psi=$ $h_{k s}(t) x_{k}^{m} z_{s}^{p}$ where $k, s, m, p=0,1,2, \ldots$ and $r_{k s}(t), g_{k s}(t), h_{k s}(t)$ are functions of $t$, respectively. It should be noted that the equation (6) could be referred to as an extended averaged equation, which is similar to the moment equations in the theory of random vibrations, where the averaging operator is taken in the probabilistic sense [10-11], [1314]. The first advantage of the Eq. (6) is that this equation is exact if the condition (5) is satisfied. The second advantage is that the Eq. (6) can be applied to arbitrary nonlinear 
systems since the condition of small nonlinearity of the system is not used for establishing the Eq. (6). Furthermore, the Eq. (6) contains both the response $Z(t)$ of the original system and the response $X(t)$ of it's corresponding homogeneous linear system. Thus, it can express links between the responses. Consequently, it allows us to make choices for the solution $Z(t)$ in some optimal ways. It seems that the averaged equation (6) may give good approximate solutions for nonlinear systems. It is noted also that the averaging procedure proposed directly to the equation (1) differs from the conventional averaging one which is performed using Lagrange variation.

The exact solution of (6) is, however, difficult to determine. One needs to solve it approximately. In order to close a set of averaged equations one needs some additional relationships between the variables. For instance, in the classical equivalent linearization and averaging methods one puts $Z(t)=X(t)$. Thus, with these methods, only a certain limited set of the equations from the hierarchy of the averaged equations can be satisfied. However, for the exact solution, all extended averaged equations have to be satisfied. One might expect that the accuracy of the technique may be better if more averaged equations are satisfied. On the other hand, $X(t)$ represents only a linear system while $Z(t)$ is from a nonlinear one. In order to satisfy more equations as well as to reduce the unknown variables one may express $Z(t)$ as a nonlinear function of $X(t): Z=G\left(X, \alpha_{1}, \alpha_{2}, \ldots \alpha_{i}, \ldots\right)$ where $\alpha_{i}$ are some unknown. parameters. Now, $Z(t)$ can express a nonlinear response properties depending on the nonlinearity level of the motion equation, which is used to be ignored when applying the above-mentioned classical methods. Which optimal form of nonlinear function $\mathrm{G}$ that can give the best approximate solution to $Z(t)$ is an open question. Since the nonlinear response is deviated from linearity, it can be expressed in a form of polynomial up to an order adequate enough to represent the original non-linear characteristics [19, 20], [13] as follows:

$$
\begin{array}{r}
z_{i}=x_{i}+\sum_{k_{1}=0}^{N} \sum_{k_{2}=0}^{N} \ldots \sum_{k_{n}=0}^{N} \alpha_{k_{1} k_{2} \ldots k_{n}} x_{1}^{k_{1}} x_{2}^{k_{2}} \ldots x_{n}^{k_{n}} \\
k_{1}+k_{2}+\ldots+k_{n} \geq 2 .
\end{array}
$$

Thus, the problem is reduced to the problem of determining $X(t)$ (or the matrix $A$ ) and the parameters $\alpha_{k_{1} k_{2} \ldots k_{n}}$. One possibility is to apply the extended averaged equations. In order to receive a closed equation system, the number of equations will be taken to be equal to the number of unknowns. Thus, the problem of solving differential equation is reduced to the problem of solving a system of algebraic equations. Which system of averaged equations is better is an open question. However, from computation and nonlinearity point of view, priority may be proposed to the functions $\psi$ with a degree as low as possible, and to the simple extended averaged equation as well as to the averaged equation depending on the nonlinearity level of the motion equation. A certain extended averaged equation system may have no solution at all or many ones. If the chosen system does not give any solution one should modify this system by changing some the original equations with other new ones. For illustration, some examples are considered. 


\section{FREE OSCILLATORS}

\subsection{Free oscillator with higher nonlinearity degree}

Consider an one-degree of freedom oscillator

$$
\left\{\begin{array}{llll}
\ddot{z}+z+\varepsilon z^{3}+\gamma z^{5}=0 & & & \text { (a) } \\
& \text { with } \quad z(0)=z_{0} & \text { (b) } \\
& \dot{z}(0)=0, & \text { (c) }
\end{array}\right.
$$

together with it's corresponding linear system

$$
\ddot{x}+k^{2} x=0 .
$$

where $k$ is unknown constant. In this case, one has

$$
Z=\{z, \dot{z}\}, X=\{x, \dot{x}\}, \quad F(Z)=\left\{\dot{z},-\left(z+\varepsilon z^{3}+\gamma z^{5}\right)\right\}, \quad p_{i}=0 .
$$

Then, the Eq. (6) takes the form

$$
\left\langle\frac{\partial \Psi}{\partial z} \dot{z}\right\rangle-\left\langle\frac{\partial \Psi}{\partial \dot{z}}\left(z+\varepsilon z^{3}+\gamma z^{5}\right)\right\rangle+\left\langle\frac{\partial \Psi}{\partial x} \dot{x}\right\rangle-\left\langle\frac{\partial \Psi}{\partial \dot{x}} k^{2} x\right\rangle=0 .
$$

Taking "the lowest" polynomial functions $\Psi(z, \dot{z}, x, \dot{x})$ from (11), one gets, for example:

$$
\begin{aligned}
& \text { for } \quad \Psi=z \dot{z} \quad\left\langle\dot{z}^{2}>-<z\left(z+\varepsilon z^{3}+\gamma z^{5}\right)>=0,\right. \\
& \text { for } \quad \Psi=x \dot{z} \quad<x\left(z+\varepsilon z^{3}+\gamma z^{5}\right)>-<\dot{z} \dot{x}>=0 \text {. }
\end{aligned}
$$

The equation (12) is conventional averaged for original variables $z, \dot{z}$ while the equation (13) contains $z, \dot{z}, x, \dot{x}$. We establish the response of the nonlinear Eq. (8) in the form

$$
z(t)=x(t)+\alpha x^{3}(t)
$$

where $x(t)$ is the solution of the Eq. (9), namely,

$$
x=a \cos \varphi, \quad \varphi=k t .
$$

For a T-period solution $z(t)$, one gets

$$
<.>=\frac{1}{T} \int_{0}^{T}(.) d t=\frac{1}{2 \pi} \int_{0}^{2 \pi}(.) d \varphi .
$$

It follows from (8.b), (15), (16)

$$
\alpha=\left(z_{0}-a\right) / a^{3} .
$$

Substituting (14)-(17) into (12), (13), after some calculations, one obtains two Eqs. for two unknowns: the amplitude a and the period $T$ (or the frequency $k$ ) of $x(t)$ as follows

$$
\begin{aligned}
& 81920 \pi^{2} a^{2}-4096 a^{2} T^{2}-448 \varepsilon a^{4} T^{2}-55 \gamma a^{6} T^{2}-98304 \pi^{2} z_{0} a-8192 z_{0} a T^{2} \\
& -1280 \varepsilon z_{0} a^{3} T^{2}-210 \gamma z_{0} a^{5} T^{2}+147456 \pi^{2} z_{0}^{2}-20480 z_{0}^{2} T^{2}-2688 \varepsilon z_{0}^{2} a^{2} T^{2} \\
& -525 \gamma z_{0}^{2} a^{4} T^{2}-5376 \varepsilon z_{0}^{3} a T^{2}-1100 \gamma z_{0}^{3} a^{3} T^{2}-14784 \varepsilon z_{0}^{4} T^{2}-2145 \gamma z_{0}^{4} a^{2} T^{2} \\
& -4290 \gamma z_{0}^{5} a T^{2}-12155 \gamma z_{0}^{6} T^{2}=0,
\end{aligned}
$$




$$
\begin{aligned}
& 16384 \pi^{2}-4096 T^{2}-384 \varepsilon a^{2} T^{2}-45 \gamma a^{4} T^{2}-40 \gamma z_{0} a^{3} T^{2}- \\
& -2688 \varepsilon z_{0}^{2} T^{2}-330 \gamma z_{0}^{2} a^{2} T^{2}-2145 \gamma z_{0}^{4} T^{2}=0,
\end{aligned}
$$

As a result, the solution $z(t)$ of the original nonlinear system (8) can be obtained from (14). The period $T_{P}$ obtained by the proposed method is compared with the exact period $T_{E}$ in the Table 1 for $z_{0}=1$ and different values of $\varepsilon$ and $\gamma$. The graphs obtained by the proposed method and by numerical simulation are presented in the Figs.1. (a-d).
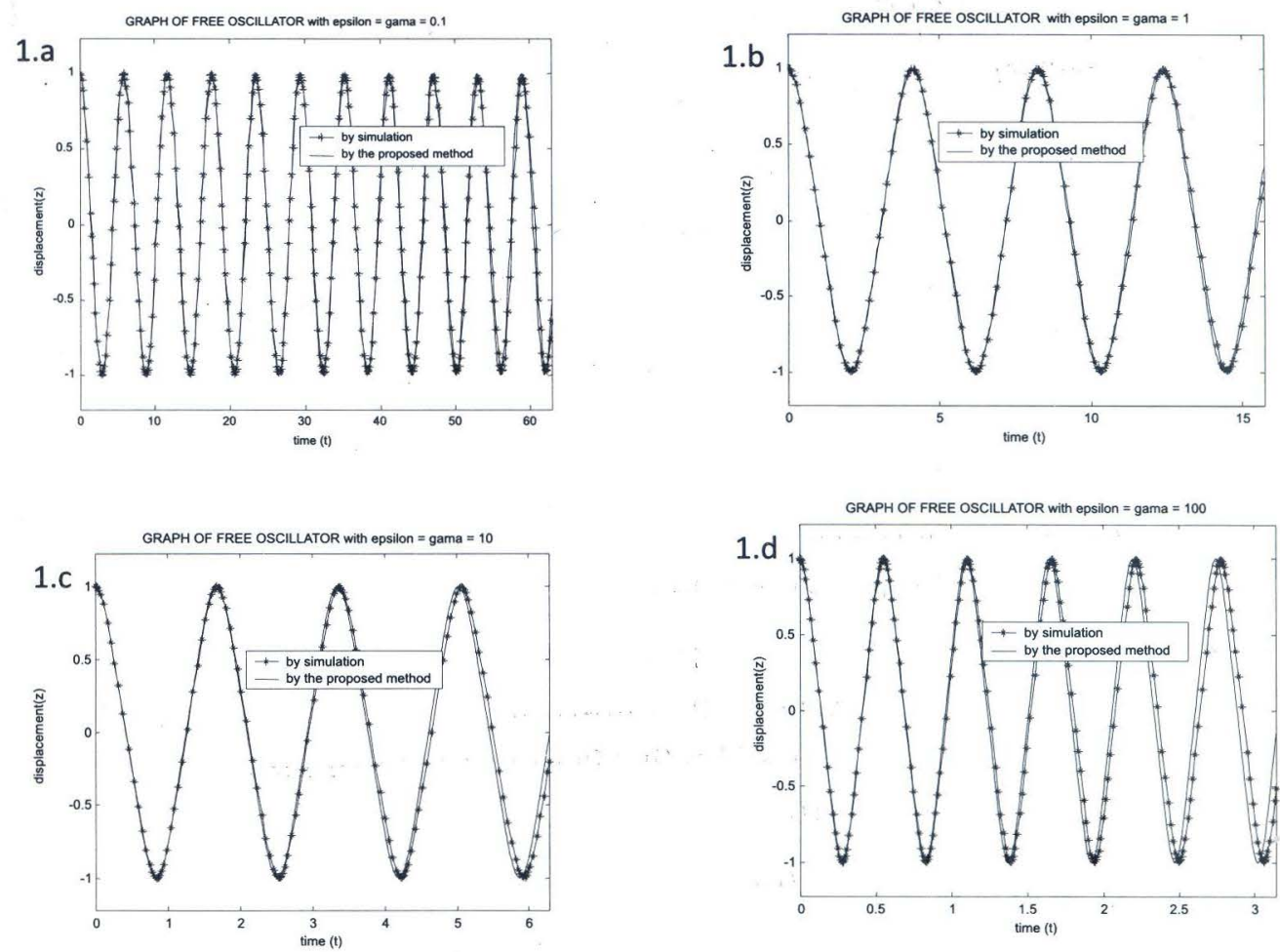

Fig. 1. Graphs of the free oscillator with higher nonlinearity with $\varepsilon=\gamma=0.1$; b. with $\varepsilon=\gamma=1$; c. with $\varepsilon=\gamma=10$; d. with $\varepsilon=\gamma=100$

Table 1. The period of free oscillation of the system 3.1

\begin{tabular}{|l|l|l|l|}
\hline$\varepsilon=\gamma$ & $\mathrm{T}_{E}$ & $\mathrm{~T}_{P}$ & error \\
\hline 0.1 & 5.9023 & 5.8912 & $0.19 \%$ \\
\hline 1 & 4.1320 & 4.1173 & $0.36 \%$ \\
\hline 10 & 1.6916 & 1.6772 & $0.85 \%$ \\
\hline 100 & 0.5545 & 0.5491 & $0.97 \%$ \\
\hline
\end{tabular}

It can be seen from the Table 1 and from the Figs. 1.(a - d) that the proposed method can give results with very high accuracy for the arbitrary values of nonlinearity. 


\section{SELF-EXCITED OSCILLATOR}

\subsection{Consider the VanderPol oscillator}

$$
\ddot{z}+z+\varepsilon\left(z^{2}-1\right) \ddot{z}=0,
$$

together with it's corresponding linear system (9). In this case, one has

$$
F(Z)=\left\{\dot{z},-\left(z+\varepsilon\left(z^{2}-1\right) \dot{z}\right)\right\}, \quad p_{i}=0 .
$$

Taking "the lowest" polynomial functions $\Psi(z, \dot{z}, x, \dot{x})$ from (11), one gets, for example:

$$
\begin{array}{ll}
\text { for } & \Psi=z \dot{z}<\dot{z}^{2}>-<z f(z, \dot{z})>=0, \\
\text { for } & \Psi=x \dot{z}<x f(z, \dot{z})>-<\dot{z} \dot{x}>=0, \\
\text { for } & \Psi=\dot{x} \dot{z}<\dot{x} f(z, \dot{z})>-k^{2}<x \dot{z}>=0, \\
\text { for } & \Psi=\dot{z}^{2} / 2<\dot{z} f(z, \dot{z})>=0,
\end{array}
$$

where

$$
f(z, \dot{z})=z+\varepsilon\left(z^{2}-1\right) \dot{z} .
$$

In order to close a set of the equations, as well as to express the non-linearity of the solution $z(t)$ of the nonlinear Eq. (20), we propose

$$
z(t)=x(t)+\alpha \dot{x}(t)+\beta x^{2}(t) \dot{x}(t),
$$

where $x(t)$ is the solution of the linear Eq. (9). Substituting (26), (27) into Eqs. (22) - (25) and using (15), (16), after some calculations, one obtains the following equations:

$$
\begin{gathered}
64 k^{2}+8 \varepsilon a^{4} k^{4} \alpha^{2} \beta-16 \varepsilon a^{2} k^{2} \beta 8 \varepsilon a^{4} k^{2} \beta+30.72 \varepsilon a^{8} k^{4} \beta^{3}+ \\
+16 \varepsilon a^{2} k^{4} \alpha^{3}+3 \varepsilon a^{6} k^{4} \alpha \beta^{2}+16 \varepsilon a^{2} k^{2} \alpha-64 \varepsilon k^{2} \alpha-64=0, \\
-5 \varepsilon a^{6} k^{2} \beta^{2}-16 a^{2} \beta-16 \varepsilon a^{2}+64 k^{2} \alpha+16 a^{2} k^{2} \beta \\
-16 \varepsilon a^{4} k^{2} \alpha \beta-16 \varepsilon a^{2} k^{2} \alpha^{2}-64 \alpha+64 \varepsilon=0, \\
8 k^{2}+5 a^{4} k^{4} \beta^{2}-a^{4} k^{2} \beta^{2}-8 k^{2} \alpha^{2} \\
+8 k^{4} \alpha^{2}+4 a^{2} k^{4} \alpha \beta-4 a^{2} k^{2} \alpha \beta-8=0, \\
272 a^{6} k^{2} \beta^{2}+128 a^{2}-512+512 a^{4} k^{2} \alpha \beta+256 a^{2} k^{2} \alpha^{2} \\
-320 a^{4} k^{2} \beta^{2}+128 a^{2} k^{4} \alpha^{4}+648^{4} k^{4} \alpha \beta^{3}+112 a^{6} k^{4} \alpha \beta^{2} \\
-256 a^{2} k^{2} \alpha \beta-512 k^{2} \alpha^{2}+13 a^{10} k^{4} \beta^{4}=0,
\end{gathered}
$$

with the 4 unknowns: the amplitude a, frequency $k$ (or the period $T$ ) of $x(t$ ), polynomial coefficients $\alpha$ and $\beta$. As a result, the solution $z(t)$ of the original nonlinear equation (20) can be obtained from (27). The oscillation amplitude $z_{P}$ and the oscillation period $T_{P}$ obtained by the proposed method are compared with the simulation quantities $z_{S}$ and $T_{S}$ in the Table 2 for different values of $\varepsilon$. 
Table 2. The period of the Vanderpol oscillation

\begin{tabular}{|c|c|c|c|c|}
\hline$\varepsilon$ & $z_{S}$ & $z_{P}$ (error) & $T_{S}$ & $T_{P}$ (error) \\
\hline 0.1 & 1.9950 & $1.9997(0.0023 \%)$ & 6.2949 & $6.2871(0.0012 \%)$ \\
\hline 1 & 2.0080 & $1.9739(0.017 \%)$ & 6.6628 & $6.4901(0.026 \%)$ \\
\hline 5 & 2.0212 & $2.0641(0.02 \%))$ & 11.4562 & $11.9872(0.046 \%)$ \\
\hline 10 & 2.0141 & $2.0982(0.04 \%)$ & 18.8690 & $19.7828(0.05 \%)$ \\
\hline
\end{tabular}
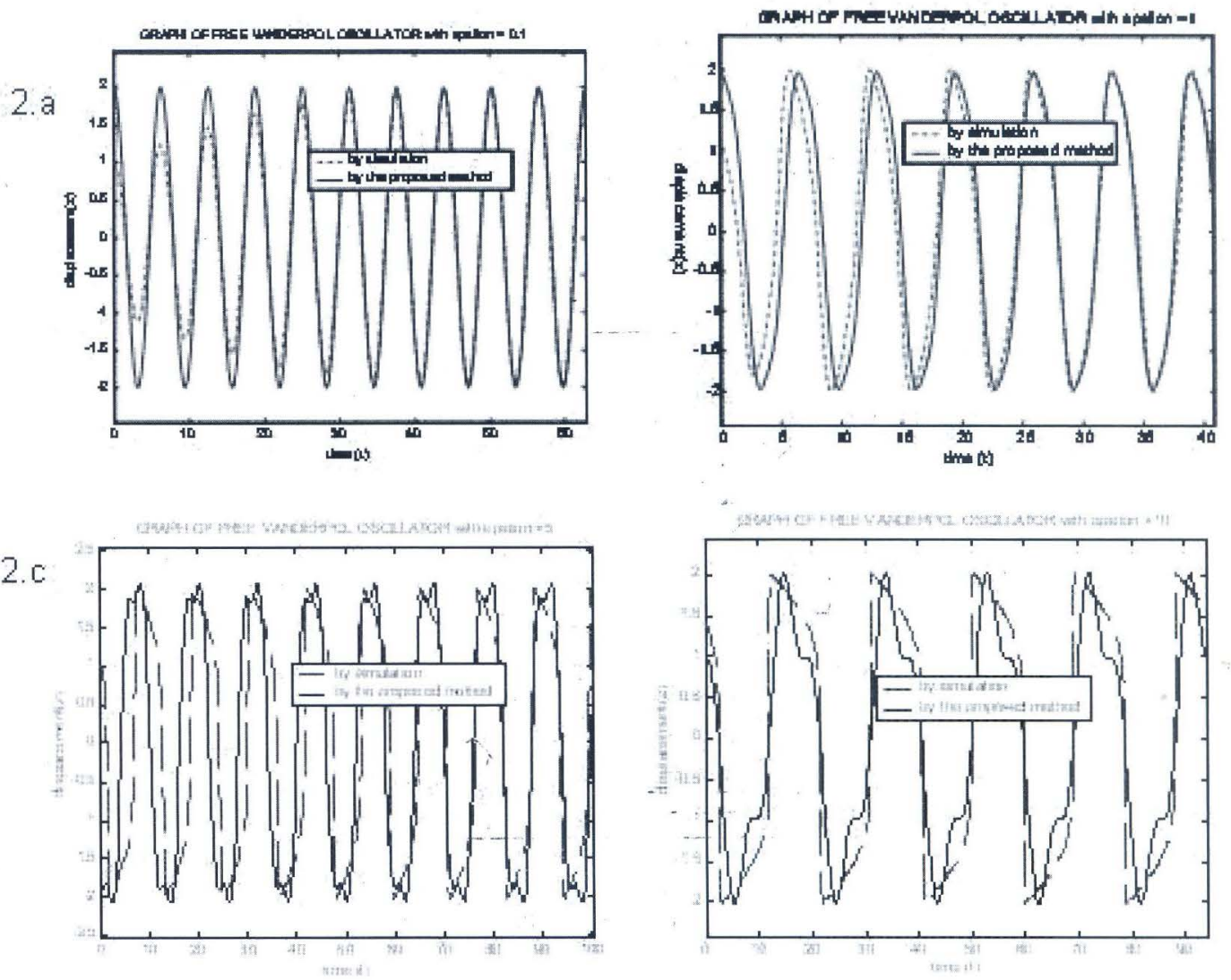

Fig. 2. Graphs of Vanderpol oscillator: a. with $\varepsilon=0.1$; b. with $\varepsilon=1$; c. with $\varepsilon$ $=5$; d. with $\varepsilon=10$

The graphs obtained by the proposed method and by numerical simulation are presented in the Figs. 2 (a-d). It can be seen from the Table 2 and from the graphs in Figs. 2(a - d) that the results of the Vanderpol oscillator obtained by the proposed method are very close to the ones obtained by numerical simulations for arbitrary values of nonlinearity. 


\section{OSCILLATION OF A NONLINEAR SUSPENSION SYSTEM}

\subsection{Freee oscillation of a nonlinear suspension system}

Consider the free oscillation of a suspension system, Mueller, Popp and Schiehlen [21], Roseau [22] which is represented schematically in Fig. 3 by two bodies of mass $m_{1}$ and $m_{2}$ linked to each other by a nonlinear spring $\left(k_{3}\right)$, a linear one $\left(k_{1}\right)$ and a shock damper with viscous damping $\left(d_{1}\right)$. Mass $m_{2}$ is contacting with the ground through a linear spring $\left(k_{2}\right)$. The non-traveling vehicle without damping $d_{1}=0$ is governed by nonlinear equations as follows

$$
\begin{aligned}
& \ddot{z}_{1}=b_{11} z_{1}+b_{12} z_{2}+b_{13} z_{1}^{3}, \\
& \ddot{z}_{2}=b_{21} z_{1}+b_{22} z_{2}+b_{23} z_{1}^{3},
\end{aligned}
$$

where it is denoted

$$
\begin{aligned}
& z_{1}=y_{1}-y_{2}, z_{2}=y_{2}, \quad \omega_{1}^{2}=\frac{k_{1}}{m_{1}}, \quad \omega_{2}^{2}=\frac{k_{2}}{m_{2}}, \quad \beta=\frac{k_{3}}{m_{1}}, b_{11}=-\omega_{1}^{2}(1+\mu), \\
& b_{12}=\omega_{2}^{2}, \quad b_{13}=-\beta(1+\mu), \quad b_{21}=\omega_{1}^{2} \mu, \quad b_{22}=-\omega_{2}^{2}, \quad b_{23}=\beta \mu, \quad \mu=\frac{m_{1}}{m_{2}} .
\end{aligned}
$$

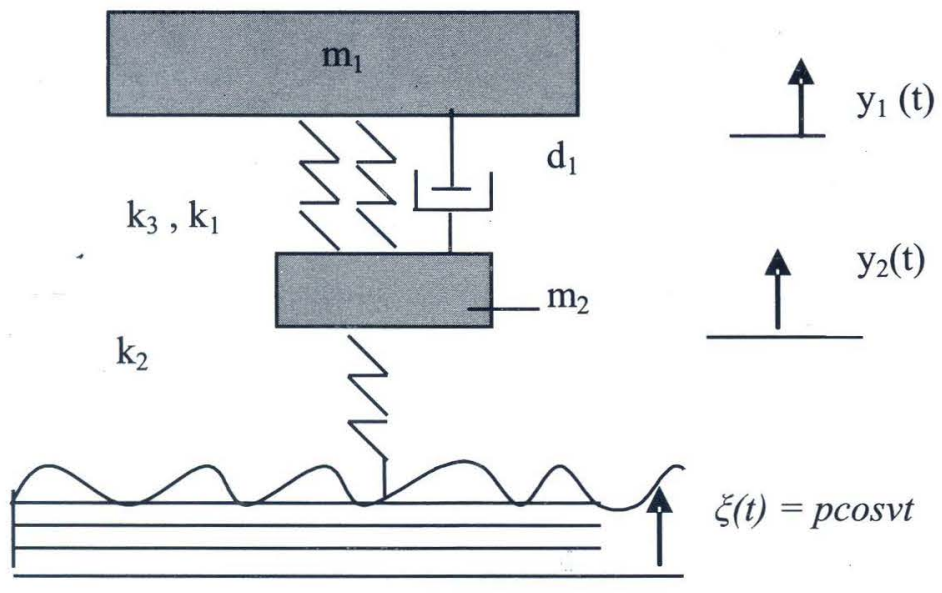

Fig. 3. Nonlinear suspension system

The linear system corresponding to the nonlinear one (32) has the form

$$
\begin{aligned}
& \ddot{x}_{1}+a_{11} x_{1}+a_{12} x_{2}=0, \\
& \ddot{x}_{2}+a_{21} x_{1}+a_{22} x_{2}=0, \\
& a_{11}=\omega_{1}^{2}(1+\mu)(1+\varepsilon), \\
& a_{12}=-\omega_{2}^{2}, \\
& a_{21}=-\omega_{1}^{2} \mu(1+\varepsilon), \\
& a_{22}=\omega_{2}^{2},
\end{aligned}
$$


where $\varepsilon$ is unknown. The general solution of the system (32) has the form

$$
\begin{aligned}
& x_{1}=c_{1} r_{1} \sin \left(\nu_{1} t+\theta_{1}\right)+c_{2} r_{2} \sin \left(\nu_{2} t+\theta_{2}\right), \\
& x_{2}=c_{1} \sin \left(\nu_{1} t+\theta_{1}\right)+c_{2} \sin \left(\nu_{2} t+\theta_{2}\right),
\end{aligned}
$$

where

$$
r_{1}=\frac{a_{12}}{\nu_{1}-a_{11}}, \quad r_{2}=\frac{a_{12}}{\nu_{2}-a_{11}} \text {. }
$$

The frequencies $\nu_{1}$ and $\nu_{2}$ are determined from the characteristic equation

$$
\left|\begin{array}{cc}
\left(a_{11}-\nu^{2}\right) & a_{12} \\
a_{21} & \left(a_{22}-\nu^{2}\right)
\end{array}\right|=0 .
$$

The constants $c_{1}, c_{2}$ and phase values $\theta_{1}, \theta_{2}$ are determined by initial conditions of the free oscillation, e.g.

$$
\begin{aligned}
& x_{1}(0)=1, \dot{x}_{1}(0)=1, \\
& x_{2}(0)=1, \dot{x}_{2}(0)=1 .
\end{aligned}
$$

The initial conditions (39) allow to represent $c_{1}, c_{2}, \nu_{1}, \nu_{2}$ as functions of $\varepsilon$. Now, one takes the response of equation (32) in the form

$$
\begin{aligned}
& z_{1}(t)=x_{1}(t)+\alpha_{1} x_{1}^{3}(t), \\
& z_{2}(t)=x_{2}(t)+\alpha_{2} x_{2}^{3}(t) .
\end{aligned}
$$

Consequently, one has got 3 unknowns $\varepsilon, \alpha_{1}, \alpha_{2}$ which can be found from the GEA equation which for an arbitrary differentiable function

$$
\Psi(X, Z)=\Psi\left(x_{1}, \dot{x}_{1}, x_{2}, \dot{x}_{2}, z_{1}, \dot{z}_{1}, z_{2}, \dot{z}_{2}\right)
$$

takes the form

$$
\begin{aligned}
& \left\langle\frac{\partial \Psi}{\partial x_{1}} \dot{x}_{1}\right\rangle+\left\langle\frac{\partial \Psi}{\partial \dot{x}_{1}} f_{1}\right\rangle+\left\langle\frac{\partial \Psi}{\partial x_{2}} \dot{x}_{2}\right\rangle+\left\langle\frac{\partial \Psi}{\partial \dot{x}_{2}} f_{2}\right\rangle \\
& +\left\langle\frac{\partial \Psi}{\partial z_{1}} \dot{z}_{1}\right\rangle+\left\langle\frac{\partial \Psi}{\partial \dot{z}_{1}} f_{3}\right\rangle+\left\langle\frac{\partial \Psi}{\partial z_{2}} \dot{z}_{2}\right\rangle+\left\langle\frac{\partial \Psi}{\partial \dot{z}_{2}} f_{4}\right\rangle=0
\end{aligned}
$$

where

$$
\begin{aligned}
f_{1} & =-\omega_{1}^{2}(1+\mu)(1+\varepsilon) x_{1}+\omega_{2}^{2} x_{2} \\
& =-a_{11} x_{1}-a_{12} x_{2}, \\
f_{2} & =\omega_{1}^{2} \mu(1+\varepsilon) x_{1}-\omega_{2}^{2} x_{2} \\
& =-a_{21} x_{1}-a_{22} x_{2}, \\
f_{3} & =-\omega_{1}^{2}(1+\mu) z_{1}+\omega_{2}^{2} z_{2}-\beta(1+\mu) z_{1}^{3} \\
& =b_{11} z_{1}+b_{12} z_{2}+b_{13} z_{1}^{3}, \\
f_{4} & =\omega_{1}^{2} \mu z_{1}-\omega_{2}^{2} z_{2}+\beta \mu z_{1}^{3} \\
& =b_{21} z_{1}+b_{22} z_{2}+b_{23} z_{1}^{3} .
\end{aligned}
$$

Taking the lowest polynomial functions $\Psi(X, Z)$ from (41), one gets: 
for $\Psi=x_{1} \dot{z}_{2}$ :

$$
<\dot{x}_{1} \dot{z}_{2}>-<x_{1} f_{4}>=0
$$

for $\Psi=x_{2} \dot{z}_{1}$ :

$$
<\dot{x}_{2} \dot{z}_{1}>-<x_{2} f_{3}>=0
$$

for $\Psi=x_{1} \dot{z}_{1}$ :

$$
<\dot{x}_{1} \dot{z}_{1}>-<x_{1} f_{3}>=0 .
$$

After some calculations, from (43-45), one obtains 3 equations $96 b_{23} c_{1}^{4} r_{1}^{4}+384 b_{23} c_{1}^{2} c_{2}^{2} r_{1}^{2} r_{2}^{2}+96 b_{23} c_{2}^{4} r_{2}^{4}+240 b_{23} c_{1}^{6} r_{1}^{6} \alpha_{1}+2160 b_{23} c_{1}^{4} c_{2}^{2} r_{1}^{4} r_{2}^{2} \alpha_{1}$ $+2160 b_{23} c_{1}^{2} c_{2}^{4} r_{1}^{2} r_{2}^{4} \alpha_{1}+240 b_{23} c_{2}^{6} r_{2}^{6} \alpha_{1}+210 b_{23} c_{1}^{8} r_{1}^{8} \alpha_{1}^{2}+3360 b_{23} c_{1}^{6} c_{2}^{2} r_{1}^{6} r_{2}^{2} \alpha_{1}^{2}+75$ $60 b_{23} c_{1}^{4} c_{2}^{4} r_{1}^{4} r_{2}^{4} \alpha_{1}^{2}+3360 b_{23} c_{1}^{2} c_{2}^{6} r_{1}^{2} r_{2}^{6} \alpha_{1}^{2}+210 b_{23} c_{2}^{8} r_{2}^{8} \alpha_{1}^{2}+63 b_{23} c_{1}^{10} r_{1}^{10} \alpha_{1}^{3}+1575$ $b_{23} c_{1}^{8} c_{2}^{2} r_{1}^{8} r_{2}^{2} \alpha_{1}^{3}+6300 b_{23} c_{1}^{6} c_{2}^{4} r_{1}^{6} r_{2}^{4} \alpha_{1}^{3}+6300 b_{23} c_{1}^{4} c_{2}^{6} r_{1}^{4} r_{2}^{6} \alpha_{1}^{3}+1575 b_{23} c_{1}^{2} c_{2}^{8} r_{1}^{2} r_{2}^{8} \alpha_{1}^{3}$ $+63 b_{23} c_{2}^{10} r_{2}^{10} \alpha_{1}^{3}+32 b_{21}\left(3 c_{1}^{4} r_{1}^{4} \alpha_{1}+4 c_{1}^{2} r_{1}^{2}\left(1+3 c_{2}^{2} r_{2}^{2} \alpha_{1}\right)+c_{2}^{2} r_{2}^{2}\left(4+3 c_{2}^{2} r_{2}^{2} \alpha_{1}\right)\right)$ $+32 b_{22}\left(3 c_{1}^{4} r_{1} \alpha_{2}+c_{2}^{2} r_{2}\left(4+3 c_{2}^{2} \alpha_{2}\right)+c_{1}^{2}\left(6 c_{2}^{2} r_{2} \alpha_{2}+r_{1}\left(4+6 c_{2}^{2} \alpha_{2}\right)\right)\right)+128 c_{1}^{2} r_{1} \nu_{1}^{2}$ $+96 c_{1}^{4} r_{1} \alpha_{2} \nu_{1}^{2}+192 c_{1}^{2} c_{2}^{2} r_{1} \alpha_{2} \nu_{1}^{2}+128 c_{2}^{2} r_{2} \nu_{2}^{2}+192 c_{1}^{2} c_{2}^{2} r_{2} \alpha_{2} \nu_{2}^{2}+96 c_{2}^{4} r_{2} \alpha_{2} \nu_{2}^{2}=0$

$96 b_{13} c_{1}^{4} r_{1}^{3}+192 b_{13} c_{1}^{2} c_{2}^{2} r_{1}^{2} r_{2}+192 b_{13} c_{1}^{2} c_{2}^{2} r_{1} r_{2}^{2}+96 b_{13} c_{2}^{4} r_{2}^{3}+240 b_{13} c_{1}^{6} r_{1}^{5} \alpha_{1}+720 b_{13} c_{1}^{6} c_{2}^{2}$ $r_{1}^{4} r_{2} \alpha_{1}+1440 b_{13} c_{1}^{4} c_{2}^{2} r_{1}^{3} r_{2}^{2} \alpha_{1}+1440 b_{13} c_{1}^{2} c_{2}^{4} r_{1}^{2} r_{2}^{3} \alpha_{1}+720 b_{13} c_{1}^{2} c_{2}^{4} r_{1} r_{2}^{4} \alpha_{1}+240 b_{13} c_{2}^{6} r_{2}^{5} \alpha_{1}$ $+210 b_{13} c_{1}^{8} r_{1}^{7} \alpha_{1}^{2}+840 b_{13} c_{1}^{6} c_{2}^{2} r_{1}^{6} r_{2} \alpha_{1}^{2}+2520 b_{13} c_{1}^{6} c_{2}^{2} r_{1}^{5} r_{2}^{2} \alpha_{1}^{2}+3780 b_{13} c_{1}^{4} c_{2}^{4} r_{1}^{4} r_{2}^{3} \alpha_{1}^{2}+37$ $80 b_{13} c_{1}^{4} c_{2}^{4} r_{1}^{3} r_{2}^{4} \alpha_{1}^{2}+2520 b_{13} c_{1}^{2} c_{2}^{6} r_{1}^{2} r_{2}^{5} \alpha_{1}^{2}+840 b_{13} c_{1}^{2} c_{2}^{6} r_{1} r_{2}^{6} \alpha_{1}^{2}+210 b_{13} c_{2}^{8} r_{2}^{7} \alpha_{1}^{2}+63 b_{13} c_{1}^{10}$ $r_{1}^{9} \alpha_{1}^{3}+315 b_{13} c_{1}^{8} c_{2}^{2} r_{1}^{8} r_{2} \alpha_{1}^{3}+1260 b_{13} c_{1}^{8} c_{2}^{2} r_{1}^{7} r_{2}^{2} \alpha_{1}^{3}+2520 b_{13} c_{1}^{6} c_{2}^{4} r_{1}^{6} r_{2}^{3} \alpha_{1}^{3}+3780 b_{13} c_{1}^{6} c_{2}^{4} r_{1}^{5}$ $r_{2}^{4} \alpha_{1}^{3}+3780 b_{13} c_{1}^{4} c_{2}^{6} r_{1}^{4} r_{2}^{5} \alpha_{1}^{3}+2520 b_{13} c_{1}^{4} c_{2}^{6} r_{1}^{3} r_{2}^{6} \alpha_{1}^{3}+1260 b_{13} c_{1}^{2} c_{2}^{8} r_{1}^{2} r_{2}^{7} \alpha_{1}^{3}+315 b_{13} c_{1}^{2} c_{2}^{8} r_{1}$ $r_{2}^{8} \alpha_{1}^{3}+63 b_{13} c_{2}^{10} r_{2}^{9} \alpha_{1}^{3}+32 b_{11}\left(3 c_{1}^{4} r_{1}^{3} \alpha_{1}+c_{2}^{2} r_{2}\left(4+3 c_{2}^{2} r_{2}^{2} \alpha_{1}\right)+2 c_{1}^{2} r_{1}\left(2+3 c_{2}^{2} r_{2}\left(r_{1}+r_{2}\right)\right.\right.$ $\left.\left.\alpha_{1}\right)\right)+32 b_{12}\left(3 c_{1}^{4} \alpha_{2}+4 c_{1}^{2}\left(1+3 c_{2}^{2} \alpha_{2}\right)+c_{2}^{2}\left(4+3 c_{2}^{2} \alpha_{2}\right)\right)+128 c_{1}^{2} r_{1} \nu_{1}^{2}+96 c_{1}^{4} r_{1}^{3} \alpha_{1} \nu_{1}^{2}$ $+192 c_{1}^{2} c_{2}^{4} r_{1} r_{2}^{2} \alpha_{1} \nu_{1}^{2}+128 c_{2}^{2} r_{2} \nu_{2}^{2}+192 c_{1}^{2} c_{2}^{2} r_{1}^{2} r_{2} \alpha_{1} \nu_{2}^{2}+96 c_{2}^{4} r_{2}^{3} \alpha_{1} \nu_{2}^{2}=0$

$96 b_{13} c_{1}^{4} r_{1}^{4}+384 b_{13} c_{1}^{2} c_{2}^{2} r_{1}^{2} r_{2}^{2}+96 b_{13} c_{2}^{4} r_{2}^{4}+240 b_{13} c_{1}^{6} r_{1}^{6} \alpha_{1}+2160 b_{13} c_{1}^{4} c_{2}^{2} r_{1}^{4} r_{2}^{2} \alpha_{1}$ $+2160 b_{13} c_{1}^{2} c_{2}^{4} r_{1}^{2} r_{2}^{4} \alpha_{1}+240 b_{13} c_{2}^{6} r_{2}^{6} \alpha_{1}+210 b_{13} c_{1}^{8} r_{1}^{8} \alpha_{1}^{2}+3360 b_{13} c_{1}^{6} c_{2}^{2} r_{1}^{6} r_{2}^{2} \alpha_{1}^{2}+75$ $60 b_{13} c_{1}^{4} c_{2}^{4} r_{1}^{4} r_{2}^{4} \alpha_{1}^{2}+3360 b_{13} c_{1}^{2} c_{2}^{6} r_{1}^{2} r_{2}^{6} \alpha_{1}^{2}+210 b_{13} c_{2}^{8} r_{2}^{8} \alpha_{1}^{2}+63 b_{13} c_{1}^{10} r_{1}^{10} \alpha_{1}^{3}+1575$ $b_{13} c_{1}^{8} c_{2}^{2} r_{1}^{8} r_{2}^{2} \alpha_{1}^{3}+6300 b_{13} c_{1}^{6} c_{2}^{4} r_{1}^{6} r_{2}^{4} \alpha_{1}^{3}+6300 b_{13} c_{1}^{4} c_{2}^{6} r_{1}^{4} r_{2}^{6} \alpha_{1}^{3}+1575 b_{13} c_{1}^{2} c_{2}^{8} r_{1}^{2} r_{2}^{8} \alpha_{1}^{3}$ $+63 b_{13} c_{2}^{10} r_{2}^{10} \alpha_{1}^{3}+32 b_{11}\left(3 c_{1}^{4} r_{1}^{4} \alpha_{1}+4 c_{1}^{2} r_{1}^{2}\left(1+3 c_{2}^{2} r_{2}^{2} \alpha_{1}\right)+c_{2}^{2} r_{2}^{2}\left(4+3 c_{2}^{2} r_{2}^{2} \alpha_{1}\right)\right)$ $+32 b_{12}\left(3 c_{1}^{4} r_{1} \alpha_{2}+c_{2}^{2} r_{2}\left(4+3 c_{2}^{2} \alpha_{2}\right)+c_{1}^{2}\left(6 c_{2}^{2} r_{2} \alpha_{2}+r_{1}\left(4+6 c_{2}^{2} \alpha_{2}\right)\right)\right)+128 c_{1}^{2} r_{1}^{2} \nu_{1}^{2}$ $+96 c_{1}^{4} r_{1}^{4} \alpha_{1} \nu_{1}^{2}+192 c_{1}^{2} c_{2}^{2} r_{1}^{2} \alpha_{1} \nu_{1}^{2}+128 c_{2}^{2} r_{2}^{2} \nu_{2}^{2}+192 c_{1}^{2} c_{2}^{2} r_{1}^{2} r_{2}^{2} \alpha_{1} \nu_{2}^{2}+96 c_{2}^{4} r_{2}^{4} \alpha_{1} \nu_{2}^{2}=0$ 
for the three unknowns $\varepsilon, \alpha_{1}, \alpha_{2}$ where $c_{1}(\varepsilon), c_{2}(\varepsilon), \nu_{1}(\varepsilon), \nu_{2}(\varepsilon)$. The system (46-48) can be solved numerically as follows:

Step 1: let $\beta=0$ (or $k_{3}=0$ ). Then, the system is linear, one obtains $\varepsilon=\alpha_{1}=\alpha_{2}=0$, in other words, $z_{1}(t) \equiv x_{1}(t) ; z_{2}(t) \equiv x_{2}(t)$.

Step 2: let $\beta=0.1$ (or $k_{3}=8$ ), the system is weak nonlinear, $\varepsilon, \alpha_{1}, \alpha_{2}$ are found in neighborhood of an initial condition $(0,0,0)$;

Step 3: let $\beta=1$ (or $k_{3}=80$ ), $\varepsilon, \alpha_{1}, \alpha_{2}$ are found in neighborhood of the initial condition $\left(\varepsilon^{0}, \alpha_{1}^{0}, \alpha_{2}^{0}\right)$, which are determined in the step 2 .

To do the same, step 3 will be applied for any other greater value up to the given one of $\beta$ (or $k_{3}$ ).

This procedure is established into a loop for software like Mathematica with "FindRoot" statement, or Matlab or Maple with "fsolve" statement, respectively. Once $\varepsilon$ is found, the linear system (43) is determined, the calculations from (37-39) allow us to find out, first $\nu_{1}, \nu_{2}$, next $r_{1}, r_{2}$ then $c_{1}, c_{2}, \theta_{1}, \theta_{2}$ and then the responses of the linear system $x_{1}(t), x_{2}(t)$. As a result, the solution $Z_{P}(t)=\left[z_{1}(t), z_{2}(t)\right]^{T}$ of the proposed method can be obtained from (40). This response $Z_{P}(t)$ is compared with a numerical simulation $Z_{S}(t)$ in the Table 3 for $m_{1}=80 \mathrm{~kg}, m_{2}=1200 \mathrm{~kg}, k_{1}=30000 \mathrm{~N} / \mathrm{m}, k_{2}=320000 \mathrm{~N} / \mathrm{m}$ and different values of $k_{3}$. The graphs obtained by the proposed method and by the numerical simulation technique are presented in the Figs. 4. $(\mathrm{a}-\mathrm{b})$, Figs. 5. $(\mathrm{a}-\mathrm{b})$.
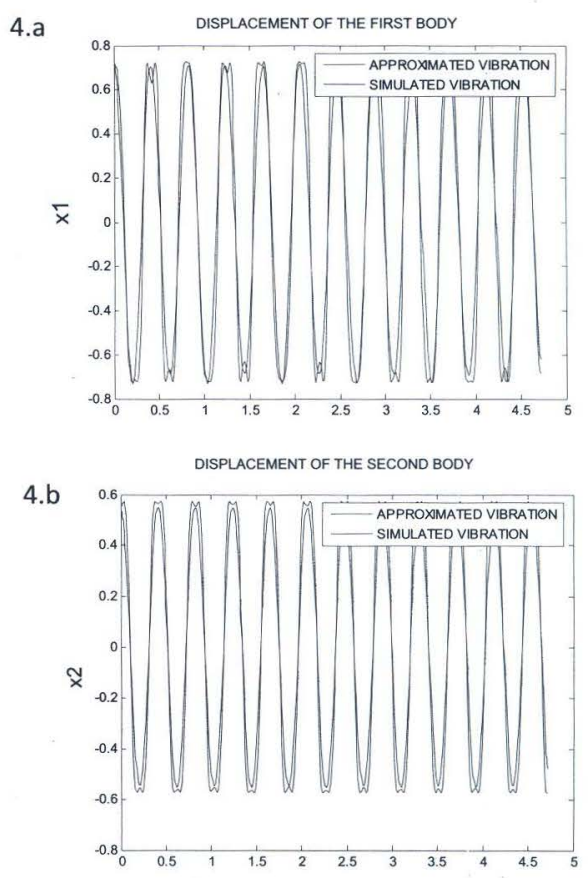

Fig. 4. Graphs of the free oscillation of the suspension system, with $\mathrm{k}_{3}=8000 \mathrm{~N} / \mathrm{m}^{3}$ 

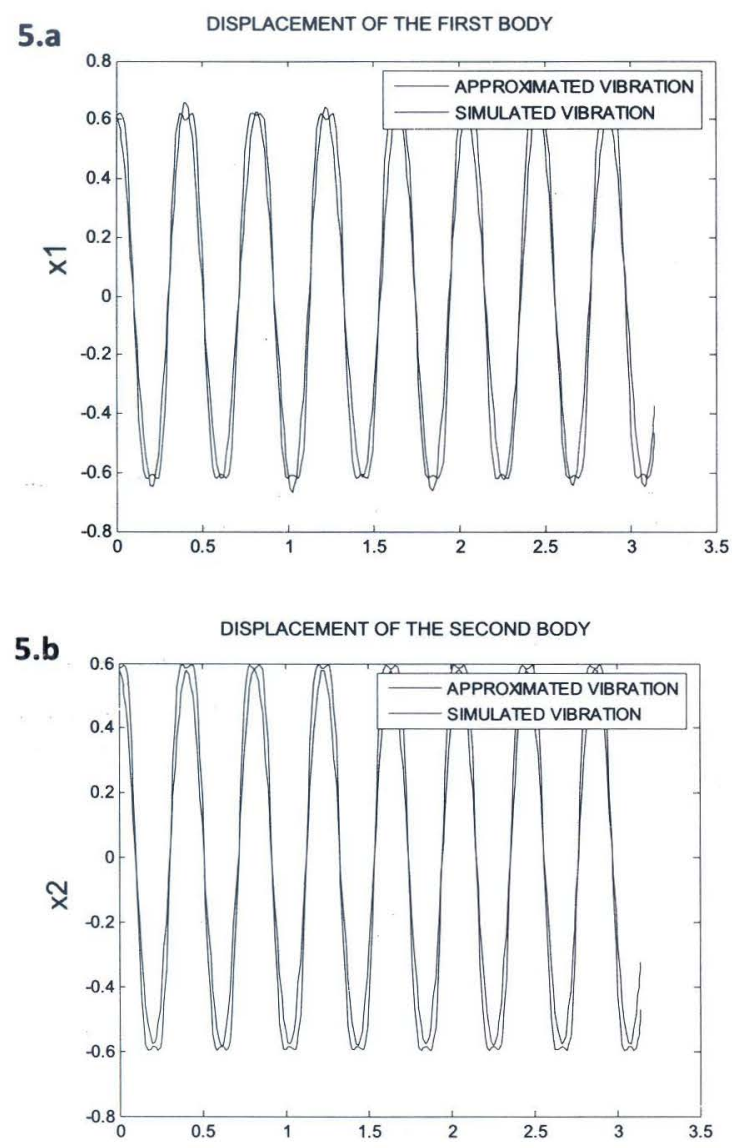

Fig. 5. Graphs of the free oscillation of the suspension system, with $\mathrm{k}_{3}=20000 \mathrm{~N} / \mathrm{m}^{3}$

It can be seen from Table 3 that the proposed method can give the oscillation amplitudes with good accuracy in comparison with the simulation. Figs.4-5 show that the frequencies obtained by the two methods are coincided in the free vibration for the system with different nonlinearity.

Table 3. Free oscillation amplitude

\begin{tabular}{|c|c|c|c|c|c|c|c|}
\hline $\mathrm{N}_{0}$ & $\mathrm{k}_{3}$ & $\mathrm{z}_{1 S} \max$ & $\mathrm{z}_{1 P \max }$ & error & $\mathrm{z}_{2 S} \max$ & $\mathrm{z}_{2 P \max }$ & error \\
\hline 1 & 80 & 1.9039 & 1.9170 & $0.68 \%$ & 1.0294 & 1.0217 & $-0.75 \%$ \\
\hline 2 & 4000 & 0.7798 & 0.7945 & $1.88 \%$ & 0.5369 & 0.5609 & $4.46 \%$ \\
\hline 3 & 8000 & 0.7273 & 0.7256 & $-0.24 \%$ & 0.5513 & 0.5728 & $3.90 \%$ \\
\hline 4 & 16000 & 0.6719 & 0.6465 & $-3.77 \%$ & 0.5755 & 0.5907 & $2.65 \%$ \\
\hline 5 & 20000 & 0.6692 & 0.6201 & $-7.34 \%$ & 0.5825 & 0.5959 & $2.30 \%$ \\
\hline
\end{tabular}




\subsection{Forced oscillation of a nonlinear suspension system}

Consider the forced periodic vibration of the suspension system shown in Fig. 3, which is governed by a differential equation system as follows

$$
\begin{aligned}
& \ddot{z}_{1}=-\omega_{1}^{2}(1+\mu) z_{1}+\omega_{2}^{2} z_{2}-\beta(1+\mu) z_{1}^{3}-\zeta(1+\mu) \dot{z}_{1}-p \cos \nu t \\
& \ddot{z}_{2}=\omega_{1}^{2} \mu z_{1}-\omega_{2}^{2} z_{2}+\beta \mu z_{1}^{3}+\zeta \mu \dot{z}_{1}+p \cos \nu t
\end{aligned}
$$

where

$$
\zeta=\frac{d_{1}}{m_{1}}, \quad p=k_{2} \frac{m_{1}+m_{2}}{2 m_{2}} .
$$

Now, for system (49), the corresponding linear one is considered

$$
\begin{aligned}
& \ddot{x}_{1}=-\omega_{1}^{2}(1+\mu) x_{1}+\omega_{2}^{2} x_{2}-\zeta(1+\mu) \dot{x}_{1}-p \cos \nu t, \\
& \ddot{x}_{2}=\omega_{1}^{2} \mu x_{1}-\omega_{2}^{2} x_{2}+\zeta \mu \dot{x}_{1}+p \cos \nu t .
\end{aligned}
$$

Firstly, the forced vibration of system (49) can be found in a form

$$
x_{1}=c_{1} \cos \nu t+s_{1} \sin \nu t, x_{2}=c_{2} \cos \nu t+s_{2} \sin \nu t .
$$

Substitution of (52) into (51), yields the following algebraic equations

$$
\begin{aligned}
& \omega_{1}^{2} s_{1}+\mu \omega_{1}^{2} s_{1}-\zeta \nu c_{1}-\mu \zeta \nu c_{1}-\nu^{2} s_{1}-\omega_{2}^{2} s_{2}=0 \\
& \omega_{2}^{2} s_{2}-\mu \zeta \nu c_{1}-\nu^{2} s_{2}-\mu \omega_{1}^{2} s_{1}=0 \\
& \zeta \nu s_{1}+\mu \zeta \nu s_{1}-\nu^{2} c_{1}+\omega_{1}^{2} c_{1}+\mu \omega_{1}^{2} c_{1}-\omega_{2}^{2} s_{2}+p=0 \\
& \omega_{2}^{2} c_{2}-\mu \zeta \nu s_{1}-\nu^{2} c_{2}-\mu \omega_{1}^{2} c_{1}-p=0
\end{aligned}
$$

Four unknowns $c_{1}, c_{2}, s_{1}, s_{2}$ can be found from (53). Then, according to (52), the solutions of the linear system $x_{1}(t)$ and $x_{2}(t)$ are determined. Now, one establishes the response of the nonlinear system (49) in the form

$$
\begin{aligned}
& z_{1}(t)=x_{1}(t)+\alpha_{1} x_{1}^{3}(t)=\left(c_{1} \cos \nu t+s_{1} \sin \nu t\right)+\alpha_{1}\left(c_{1} \cos \nu t+s_{1} \sin \nu t\right)^{3}, \\
& z_{2}(t)=x_{2}(t)+\alpha_{2} x_{2}^{3}(t)=\left(c_{2} \cos \nu t+s_{2} \sin \nu t\right)+\alpha_{2}\left(c_{2} \cos \nu t+s_{2} \sin \nu t\right)^{3},
\end{aligned}
$$

where, $\alpha_{1}, \alpha_{2}$ are to be found from the GEA equation (41), with

$$
\begin{aligned}
& f_{1}=-\omega_{1}^{2}(1+\mu) x_{1}+\omega_{2}^{2} x_{2}-\zeta(1+\mu) \dot{x}_{1}-p \cos \nu t \\
& f_{2}=\omega_{1}^{2} \mu x_{1}-\omega_{2}^{2} x_{2}+\zeta \mu \dot{x}_{1}+p \cos \nu t \\
& f_{3}=-\omega_{1}^{2}(1+\mu) z_{1}+\omega_{2}^{2} z_{2}-\beta(1+\mu) z_{1}^{3}-\zeta(1+\mu) \dot{z}_{1}-p \cos \nu t \\
& f_{4}=\omega_{1}^{2} \mu z_{1}-\omega_{2}^{2} z_{2}+\beta \mu z_{1}^{3}+\zeta \mu \dot{z}_{1}+p \cos \nu t .
\end{aligned}
$$

Taking the lowest polynomial functions $\Psi(X, Z)$ from (41), one gets

$$
\begin{aligned}
& \text { for } \quad \Psi=x_{2} \dot{z}_{1}<\dot{x}_{2} \dot{z}_{1}>-<x_{2} f_{3}>=0, \\
& \text { for } \quad \Psi=x_{1} \dot{z}_{2} \quad<\dot{x}_{1} \dot{z}_{2}>-<x_{1} f_{4}>=0 .
\end{aligned}
$$


Substitution of (52), (54), (55) into (56), (57), after some calculations, one obtains two equations for two unknowns $\alpha_{1}, \alpha_{2}$, as follows:

$$
\begin{aligned}
& 3 p^{4}\left(21 p^{6} \alpha_{1}^{3} \beta(1+\mu) \nu^{18}\left(\nu^{2}-\omega_{1}^{2}\right)+70 p^{4} \alpha_{1}^{2} \beta(1+\mu) \nu^{14}\left(\nu^{2}-\omega_{1}^{2}\right)\left(\zeta^{2}\right.\right. \\
& \left.\left((1+\mu) \nu^{3}-\nu \omega_{2}^{2}\right)^{2}+\left(\nu^{4}+\omega_{1}^{2} \omega_{2}^{2}-\nu^{2}\left((1+\mu) \omega_{1}^{2}+\omega_{2}^{2}\right)\right)^{2}\right)+80 p^{2} \alpha_{1} \beta \\
& (1+\mu) \nu^{10}\left(\nu^{2}-\omega_{1}^{2}\right)\left(\zeta^{2}\left((1+\mu) \nu^{3}-\nu \omega_{2}^{2}\right)^{2}+\left(\nu^{4}+\omega_{1}^{2} \omega_{2}^{2}-\nu^{2}((1+\mu)\right.\right. \\
& \left.\left.\left.\omega_{1}^{2}+\omega_{2}^{2}\right)\right)^{2}\right)^{2}+32\left(\beta(1+\mu) \nu^{6}\left(\nu^{2}-\omega_{1}^{2}\right)-\alpha_{1} \nu^{6}\left(\zeta^{2}(1+\mu) \nu^{2}+\left(\nu^{2}\right.\right.\right. \\
& \left.\left.\left.-\omega_{1}^{2}\right)\left(\nu^{2}-(1+\mu) \omega_{1}^{2}\right)\right)+\alpha_{2}\left(\zeta^{2} \nu^{2}+\left(\nu^{2}-\omega_{1}^{2}\right)^{2}\right)^{2} \omega_{2}^{2}\right)\left(\zeta ^ { 2 } \left((1+\mu) \nu^{3}\right.\right. \\
& \left.\left.\left.-\nu \omega_{2}^{2}\right)^{2}+\left(\nu^{4}+\omega_{1}^{2} \omega_{2}^{2}-\nu^{2}\left((1+\mu) \omega_{1}^{2}+\omega_{2}^{2}\right)\right)^{2}\right)^{3}\right)=0 \\
& 3 p^{4} \nu^{2}\left(21 p^{6} \alpha_{1}^{3} \beta \mu \nu^{18}+70 p^{4} \alpha_{1}^{2} \beta \mu \nu^{14}\left(\zeta^{2}\left((1+\mu) \nu^{3}-\nu \omega_{2}^{2}\right)^{2}+\left(\nu^{4}\right.\right.\right. \\
& \left.\left.+\omega_{1}^{2} \omega_{2}^{2}-\nu^{2}\left((1+\mu) \omega_{1}^{2}+\omega_{2}^{2}\right)\right)^{2}\right)+80 p^{2} \alpha_{1} \beta \mu \nu^{10}\left(\zeta^{2}\left((1+\mu) \nu^{3}-\nu \omega_{2}^{2}\right)^{2}\right. \\
& \left.+\left(\nu^{4}+\omega_{1}^{2} \omega_{2}^{2}-\nu^{2}\left((1+\mu) \omega_{1}^{2}+\omega_{2}^{2}\right)\right)^{2}\right)^{2}-32\left(-\mu \nu^{6}\left(\beta+\alpha \omega_{1}^{2}\right)+\alpha_{2}\right. \\
& \left.\left(\nu^{2}-\omega_{1}^{2}\right)\left(\zeta^{2} \nu^{2}+\left(\nu^{2}-\omega_{1}^{2}\right)^{2}\right)\left(\nu^{2}-\omega_{2}^{2}\right)\right)\left(\zeta^{2}\left((1+\mu) \nu^{3}-\nu \omega_{2}^{2}\right)^{2}+\left(\nu^{4}\right.\right. \\
& \left.\left.\left.+\omega_{1}^{2} \omega_{2}^{2}-\nu^{2}\left((1+\mu) \omega_{1}^{2}+\omega_{2}^{2}\right)\right)^{2}\right)^{3}\right)=0
\end{aligned}
$$

The amplitude of $Z_{P}(t)$ and the maximum of a numerical simulation response $Z_{S}(t)$ in stability stage are compared in Table 2 for $m_{1}=80 \mathrm{~kg}, m_{2}=1200 \mathrm{~kg}, k_{1}=30000$ $\mathrm{N} / \mathrm{m}, k_{2}=320000 \mathrm{~N} / \mathrm{m}, d_{1}=4800 \mathrm{Ns} / \mathrm{m}, \nu=2 \pi 1 / \mathrm{s}$ and different values of $k_{3}$.

Table 4. Forced oscillation amplitude

\begin{tabular}{|c|c|c|c|c|c|c|c|}
\hline $\mathrm{N}_{0}$ & $\mathrm{k}_{3}$ & $\mathrm{z}_{1 S} \max$ & $\mathrm{z}_{1 P \max }$ & error & $\mathrm{z}_{2 S} \max$ & $\mathrm{z}_{2 P \max }$ & error \\
\hline 1 & 80 & 5.5717 & 5.6994 & $2.24 \%$ & 75.6614 & 76.0461 & $0.51 \%$ \\
\hline 2 & 800 & 4.8015 & 4.5172 & $-5.92 \%$ & 75.9515 & 76.0369 & $0.11 \%$ \\
\hline 3 & 4000 & 3.3624 & 3.2369 & $-3.73 \%$ & 76.3699 & 76.0249 & $-0.45 \%$ \\
\hline 4 & 8000 & 3.0027 & 2.9031 & $-3.32 \%$ & 74.5263 & 76.0192 & $2.01 \%$ \\
\hline 5 & 16000 & 2.5791 & 2.6512 & $2.79 \%$ & 73.3357 & 76.0135 & $3.65 \%$ \\
\hline
\end{tabular}

It is seen from Table 4 that the proposed method can give results with very high accuracy for arbitrary values of nonlinearity.

\section{CONCLUSIONS}

The method introduces the so-called extended averaged equations involving the variables of the original nonlinear and of the corresponding linear systems. Furthermore, the technique proposes to represent a periodic solution of nonlinear systems by a polynomial of harmonic solution of its corresponding linear systems. Thus, a possible way to determine the solution polynomial coefficients and the linear system can be derived. The technique is quite simple since it can use properties of harmonic functions although the calculations are more complicated than the averaging method.

The proposed method can be applied to both stochastic oscillations and deterministic oscillations. The extended averaged equation is established not using the condition 
of small nonlinearity. Thus, the method can be applied to arbitrarily nonlinear systems. The corresponding linear system is essential for this methodology. The term "corresponding" means the nonlinear and linear systems have the same dimension, i.e, the numbers of components of the vectors $\mathrm{Z}$ and $\mathrm{X}$ are equal to each other. Furthermore, the linear system should have expected properties of the nonlinear system. For illustration, for illustration, some free/self-excited oscillators and nonlinear free/ forced oscillations of a suspension system with two-degree-of-freedom are investigated. The numerical results give good approximate solutions for the system with the quite large range of the values of the nonlinearity coefficient. However, the technique should be tested for other nonlinear systems, for example, for systems with unsymmetry properties. Some related questions on the set of extended averaged equations and the form of polynomials to be chosen to get a better approximate solution have to be discussed.

\section{REFERENCES}

[1] A. H. Nayfeh, Perturbation Methods, John Wiley \& sons, 1973.

[2] N. N. Bogoliubov, Yu. A. Mitropolskii, Asymptotic methods in the theory of nonlinear oscillations, $4^{\text {th }}$ ed., Moscow, 1974.

[3] A. H. Nayfeh, and D. T. Mook, Nonlinear Oscillations, John Wiley \& sons, 1979.

[4] G. Schmidt, A. Tondl, Nonlinear Vibration, Cambridge University Press, 1986.

[5] M. Roseau, Vibrations in Mechanical Systems, Springer Verlag, 1989.

[6] S. Wiggins, Introduction to Applied Nonlinear Dynamical Systems and Chaos, SpringerVerlag, NewYork, 1990.

[7] Yu. A. Mitropolskii, Nguyen Van Dao, Nguyen Dong Anh, Nonlinear oscillations in the systems of arbitrary order, "Naukova" (Science), Kiev, 1992 (in Russian).

[8] A. H. Nayfeh, and B. Balachandran, Applied Nonlinear Dynamics, Wiley, NewYork, 1995.

[9] Lawrence, N. Virgin, Introduction to Experimental Nonlinear Dynamics, A Case Study in Mechanical Vibration, Cambridge University press, 2000.

[10] J. B. Roberts, and P. D. Spanos, Random Vibration and Stochastic Linearization, John Wiley and Sons, 1990.

[11] N. D. Anh, W. Schiehlen, An approach to the problem of closure in the non- linear stochastic Mechanics, Int. J. of Mechanics 29 (1994) 109 - 123.

[12] S. F. Wojtkiewicz, B. F. Spencer, and L. A. Bergman, On the cumulant-neglect closure method in stochastic Dynamics, Int. J. Non-Linear Mechanics 31 (1996) (2) 657- 684.

[13] N. D. Anh, N. Q. Hai, A Technique of Closure Using a Polynomial Function of Gaussian Process, Probabilistic Engineering Mechanics 15(2000) 191-197, Elsevier Science Ltd.

[14] N. D. Anh, N. Q. Hai, A Technique for Solving Nonlinear Systems Subject to Random Excitation, IUTAM Symposium on Recent Developments in Non-linear Oscillations of Mechanical Systems, 217-226, Kluwer Academic Publishers, 2000.

[15] J. A. Sanders, and F. Verhulst, Averaging Methods in Nonlinear Dynamical Systems, Springer-Verlag, NewYork, 1985.

[16] Jack Hale, Theory of Functionnal Differential Equations, Springer, Verlag, Newyork, Heidelberg, Berlin 1977.

[17] R. V. Roy, Averaging Method for Strongly Nonlinear Oscillations with Periodic Excitation, International Journal of Nonlinear Mechanics 29, 737-753, 1994.

[18] V. N. Bogaevsky, A. YA. Povzner, Algebraic methods in the non-linear theory of pertubations," Nauka " (Science), Moscow, 1987 (in Russian) 
[19] M. Grigoriu, Applied Non-Gaussian Processes, PTR Prentice Hall, Englewood Cliffs, NJ, 1995.

[20] L. D. Lutes, and S. Sarkani, Stochastic Analysis of Structural and Mechanics, Prentice-Hall, . Englewood Cliffs, NJ, 1997.

[21] P. C. Mueller, K. Popp, W. O. Schiehlen, Berechnungsverfahren stochastischer Fahrzengschwingungen, Ingenieur -Archiv 49 (1980) 235-254.

[22] M. Roseau, Vibrations in Mechanical Systems, Berlin Springer Verlag, 1989.

Received May 19, 2009

\section{PHƯƠNG PHÁP PHƯƠNG TRÌNH TRUNG BİNH HOÁ SUY RỘNG VÀ ỬNG DỤNG TRONG VIỆC PHÂN TÍCH MộT SỐ HỆ DAO ĐỘNG PHI TUYẾN}

Bài báo trình bày phương pháp phương trình trung bình hoá suy rộng trong việc nghiên cứu bài toán dao động phi tuyến. Phương pháp đề xuất được áp dụng cho một số hệ dao động tự do/ tự dao động phi tuyên một bậc tự do cũng như dao động tự do/ cưỡng bức của hệ treo phi tuyến hai bậc tự do. Các kết quả trong việc phân tích các hệ dao động này với mức độ phi tuyến khác nhau đã chỉ ra tính hiệu quả và những ưu điểm của phương pháp này. 